\title{
Two fluid acoustic modes and inhomogeneous cosmologies
}

\author{
J P Krisch $\dagger$ and L L Smalley \\ † Department of Physics, University of Michigan, Ann Arbor, MI 48109, USA \\ ‡ Department of Physic, University of Alabama in Huntsville, Huntsville, AL 35899, USA
}

Received 10 June 1993, in final form 27 July 1993

\begin{abstract}
The weak discontinuities that can propagate in a relativistic two fluid system are examined and expressions obtained for their speed of propagation. The acoustic speeds and discontinuity amplitudes are described by their Frenet parameters. Both longitudinal and transverse acoustic modes are possible. The discontinuity in the two fluid anisotropy vector can be timelike, spacelike, or null. As an application we use the existence of two longitudinal modes to extend the fluid content of the inhomogeneous cosmologies discussed by Van den Bergh and Skea. For their solution we find a family of two perfect fluids whose properties range from a dust-perfect-fluid set to two $\gamma=\frac{4}{3}$ comoving perfect fluids.
\end{abstract}

PACS numbers: $0420,0440,0340$

\section{Introduction}

Two fluid systems have a stress-energy content of the form:

$$
T^{\mu \nu}=\left(\varepsilon_{1}+p_{1}\right) U^{\mu}{ }_{1} U^{\nu}{ }_{1}+\left(\varepsilon_{2}+p_{2}\right) W^{\mu}{ }_{2} W^{\nu}{ }_{2}+\left(p_{1}+p_{2}\right) g^{\mu \nu},
$$

where $U^{\mu}{ }_{1}$ and $W^{\prime \prime}{ }_{2}$ are the physical fiuid velocities and $U^{\mu}{ }_{1} U_{\mu 1}=-1, W^{\mu}{ }_{2} W_{\mu_{2}}=-1$; $\mu$ is the spacetime index; $(1,2)$ labels the physical velocity. Two fluids can be a convenient stress-energy model for many different physical situations. Recently, for example, two fluid models have been actively applied to cosmological models in which one fluid represents radiation and the second a matter content for the universe [1-9]. If the two fluids are assumed to be comoving, then the stress-energy is effectively that of a single fluid:

$$
T^{\mu \nu}=(\varepsilon+p) U^{\mu} U^{\nu}+p g^{\mu \nu},
$$

with $\varepsilon=\varepsilon_{1}+\varepsilon_{2}$ and $p=p_{1}+p_{2}$. If the fluids are not comoving, then Letelier [10] has shown that equation (1) can be transformed into a tensor explictly exhibiting a preferred spatial direction:

$$
T^{\mu \nu}=\varepsilon U^{\mu} U^{\nu}+\pi\left(g^{\mu \nu}+U^{\mu} U^{\nu}\right)+(\sigma-\pi) W^{\mu} W^{\nu},
$$

where $\varepsilon$ is the effective energy density and $\pi$ and $\sigma$ are anisotropic pressures. $U^{\mu}$ is a timelike unit vector and $W^{\mu}$ a spacelike unit vector, both obtained from the physical velocities by a rotation. The relation between these fluid parameters and the physical fluid parameters is reviewed in section 4 of this paper. At first glance this stress-energy tensor looks like that of a viscous fluid. It is important to realize that $W^{\mu}$ is independent of $U^{\mu}$, 
and represents the direction of anisotropy in the medium, related to the motion of one fluid relative to the other.

In this note we examine some of the sound waves that can propagate in a relativistic two fluid system. In addition to their intrinsic interest, the speeds of some of the waves are useful in interpreting equations of state for relativistic two fluid models. In section 2 we consider the conservation laws and obtain the wave speeds. Section 3 contains a discussion of some of the acoustic modes. In section 4 we use the existence of two longitudinal fluid modes to extend an inhomogeneous perfect fluid cosmology discussed by Van den Bergh and Skea [11].

\section{Conservation laws and wave speeds}

\subsection{Conservation laws}

The conservation laws associated with equation (3) are:

$$
T_{; \nu}^{\mu \nu}=0,
$$

or, on rewriting:

$$
\begin{aligned}
\varepsilon_{, \nu} U^{\mu} U^{\mu}+ & (\pi+\varepsilon)\left(U_{i \nu}^{\mu} U^{\nu}+U^{\mu} U_{; \nu}^{\nu}\right)+\pi_{\nu \nu}\left(g^{\mu \nu}+U^{\mu} U^{\nu}\right) \\
& +(\sigma-\pi)_{, \nu} W^{\mu} W^{\nu}+(\sigma-\pi)\left(W^{\mu}{ }_{i \nu} W^{\nu}+W^{\mu} W^{\nu}{ }_{\nu \nu}\right)=0 .
\end{aligned}
$$

The projection along the timelike $U^{\mu}$ is:

$$
\varepsilon_{; \nu} U^{\nu}+U^{\nu}{ }_{i \nu}(\varepsilon+\pi)-(\sigma-\pi) U^{\mu} W^{\nu} W_{\mu ; \nu}=0 .
$$

Using the projection operator $g_{\mu \nu}+U_{\mu} U_{\nu}$, the spatial projection is:

$$
\begin{aligned}
& (\varepsilon+\pi) U_{\mu: \nu} U^{\nu}+\pi_{, \mu}+U_{\mu} \pi_{, \nu} U^{\nu}+(\sigma-\pi)_{. \nu} W^{\nu} W_{\mu} \\
& +(\sigma-\pi)\left(W_{\mu: \nu} W^{\nu}-U_{\mu} W^{\nu} U_{\nu ; \alpha} W^{\alpha}+W_{\mu} W_{; \nu}^{\nu}\right)=0,
\end{aligned}
$$

where we used the othogonality of $U^{\mu}$ and $W^{\mu}$ to switch covariant derivatives in the last line of equation (7).

\subsection{Discontinuities}

The wavefront that we will consider is a hypersurface across which the accelerations $\dot{U}^{\mu}=U^{\mu}{ }_{i \nu} U^{\nu}$ and $\dot{W}^{\mu}=W^{\mu}{ }_{; \nu} U^{\nu}$ are discontinuous. The propagation of this surface through the medium can be described by a set of unit vectors $i^{\mu}, V^{\mu}$, and $\lambda^{\mu}$, in the notation of Carter [12]. $V^{\mu}$ is the vector describing the direction of propagation. $i^{\mu}$ is the polarization vector describing the relation of the acceleration discontinuity in $U^{\mu}$ to the direction of propagation. We shall later identify the polarization vector with a particular member of the Frenet triad. $\lambda^{\mu}$ is the normal to the characteristic hypersurface associated with the wavefront. We have:

$$
\begin{aligned}
& \lambda^{\mu}=V^{\mu}-w U^{\mu}, \\
& U^{\mu} i_{\mu}=U^{\mu} V_{\mu}=0,
\end{aligned}
$$

where $w$ is the velocity of wave propagation relative to the fluid flow [13]. 
The method that we shall use to discuss the wave propagation is Hadamard's method as applied by Carter [12] to the propagation of sound in high pressure relativistic solids. The method assumes that the discontinuities of the fluid functions across the wavefront hypersurface are proportional to the normal, $\lambda^{\mu}$, to the hypersurface, i.e., that we are dealing with weak discontinuities in the field functions [14]. Denoting the discontinuity by square brackets, we can write:

$$
\begin{array}{lc}
{\left[\varepsilon_{, \mu}\right]=\underline{\varepsilon} \lambda_{\mu}} & {\left[\pi_{, \mu}\right]=\underline{\pi \varepsilon} \lambda_{\mu} \quad\left[\sigma_{, \mu}\right]=\underline{\sigma \varepsilon \lambda_{\mu}}} \\
{\left[U^{\nu}{ }_{; \mu}\right]=\underline{U}^{v} \lambda_{\mu}} & {\left[W^{\nu}{ }_{; \mu}\right]=\underline{W}^{v} \lambda_{\mu}}
\end{array}
$$

where the underlined quantities are the proportionality functions. We have assumed that the pressure changes are driven by the density changes. Non-relativistically we would write, for example, $\underline{\pi \varepsilon}=(\delta \pi / \delta \varepsilon) \mathrm{d} \varepsilon$. The thermodynamics of a mixed system can be very complex, possibly involving the entropy of mixing. We will consider only isentropic processes.

The discontinuities in $U^{\mu}$ and $W^{\mu}$ defined by equation (9) can be rewritten in a very convenient form by using a Frenet tetrad to describe the relationship between $U^{\mu}$ and $W^{\mu}$. The Frenet tetrad $\left(U^{\mu}, A^{\mu}, B^{\mu}, C^{\mu}\right)$ is assumed to have a timelike member along the fluid velocity.

For the tetrad we have [15]:

$$
\left[\begin{array}{c}
\dot{U}^{\mu} \\
\dot{A}^{\mu} \\
\dot{B}^{\mu} \\
\dot{C}^{\mu}
\end{array}\right]=\left[\begin{array}{cccc}
0 & \kappa & 0 & 0 \\
\kappa & 0 & \tau_{1} & 0 \\
0 & -\tau_{1} & 0 & \tau_{2} \\
0 & 0 & -\tau_{2} & 0
\end{array}\right]\left[\begin{array}{l}
U^{\mu} \\
A^{\mu} \\
B^{\mu} \\
C^{\mu}
\end{array}\right]
$$

with $\kappa$ the curvature, and $\tau_{1}$ and $\tau_{2}$ the first and second torsions associated with the fluid trajectory. The acceleration vector lies along $A^{\mu}$ :

$$
\dot{U}^{\mu}=\kappa A^{\mu}
$$

so that the discontinuity across the front is:

$$
\left[\dot{U}^{\mu}\right]=[\kappa] A^{\mu} \text {. }
$$

Comparing with equation (9), we find that:

$$
w \underline{U}^{\mu}=[\kappa] A^{\mu} \text {. }
$$

$[\kappa]$, the discontinuity in the curvature can be identified with Carter's [12] $\alpha$, the amplitude of the acceleration jump. With this choice of basis tetrad, the polarization vector $i^{\mu}$ is $A^{\mu}$. The propagation vector $V^{\mu}$ can be any of the spacelike triad $\left(A^{\mu}, B^{\mu}, C^{\mu}\right)$. The discontinuities in $W^{\mu}$ can be treated in the same way as those in $U^{\mu}$. Since $W^{\mu}$ can lie along any of the spacelike triad $\left(A^{\mu}, B^{\mu}, C^{\mu}\right)$, we have, using equations (8)-(10):

$$
w \underline{W}^{\mu}=\left[W_{; v}^{\mu}\right] U^{\nu} ;
$$

if $W^{\mu}=A^{\mu}$, then:

$$
w \underline{W}^{\mu}=[\kappa] U^{\mu}+\left[\tau_{1}\right] B^{\mu} ;
$$

if $W^{\mu}=b^{\mu}$, then:

$$
w \underline{W}^{\mu}=-\left[\tau_{1}\right] A^{\mu}+\left[\tau_{2}\right] C^{\mu}
$$

if $W^{\mu}=C^{\mu}$, then:

$$
w \underline{W^{\mu}}=-\left[\tau_{2}\right] B^{\mu}
$$

where $\left[\tau_{1}\right]$ and $\left[\tau_{2}\right]$ represent the jump discontinuities in the first and second torsion. They will contribute to the amplitude of the discontinuity in $W^{\mu}$ along the respective directions and are analogous to Carter's [12] $\alpha$ for the amplitude of the discontinuity in the fluid's acceleration. We have identified $\alpha$ with $[k]$. 


\subsection{Discontinuities and conservation}

Taking the discontinuities of equation (6) and using (15)-(17) one finds:

$$
w^{2} \underline{\varepsilon}=-[\kappa]\left\{(\varepsilon+\pi) A^{\mu} V_{\mu}+(\sigma-\pi) W^{\mu} V_{\mu} W^{v} A_{\nu}\right\} .
$$

The discontinuities in the spatial projections of the conservations laws, equation (7), are:

$$
\begin{aligned}
{[\kappa](\varepsilon+\pi) A^{\mu} } & +\underline{\pi \varepsilon} V^{\mu}+(\sigma-\underline{\pi}) \varepsilon V^{\nu} W_{\nu} W^{\mu}+(\sigma-\pi)\left\{\underline{W}^{\mu} V^{\mu} W_{\nu}\right. \\
& \left.-[\kappa] w^{-1} A^{\nu} W_{\nu} V^{\alpha} W_{\alpha} U^{\mu}\right\}+(\sigma-\pi)\left\{W^{\mu} V^{\mu} \underline{W}_{\nu}+[\kappa] A^{\nu} W_{\nu} W^{\mu}\right\}=0 .
\end{aligned}
$$

There are three choices for $W^{\mu}$, and we shall consider them separately.

2.3.1. $W^{\mu}=A^{\mu}$. Using equation (15) to describe $W \mu$, we find for equation (18):

$$
w^{2} \underline{\varepsilon}=-(\varepsilon+\sigma)[\kappa] V^{v} A_{v},
$$

where we have used $W^{\mu}=A^{\mu}$, and for equation (19):

$$
\begin{aligned}
A^{\mu}\{[\kappa](\sigma+\varepsilon) & \left.+\underline{\varepsilon}(\sigma-\underline{\pi}) V^{\nu} A_{\nu}+(\sigma-\pi) w^{-1}\left[\tau_{1}\right] V^{\nu} B_{\nu}\right\} \\
& +\underline{\pi \varepsilon V^{\mu}}+(\sigma-\pi) w^{-1}\left[\tau_{1}\right] V^{\nu} A_{\nu} B^{\mu}=0 .
\end{aligned}
$$

If the wave is propagating along $A^{\mu}, V^{\mu}$ is parallel to $A^{\mu}$, and we have:

$$
A^{\mu}\{[\kappa](\varepsilon+\sigma)+\underline{\sigma \varepsilon}\}+B^{\mu}(\sigma-\pi)\left[\tau_{1}\right] w^{-1}=0 .
$$

The projection along $A^{\mu}$, along with (18), gives the first acoustic mode:

$$
w_{1}=\sqrt{\underline{\sigma}} \quad V^{\mu} A_{\mu}=1, W^{\mu}=A^{\mu} .
$$

From the $B^{\mu}$ projection we see that there is no discontinuity in the first torsion, $\left[\tau_{1}\right]=0$.

If the propagation is not along $A^{\mu}$, then there is no density variation, $\underline{\varepsilon}=0$, and equation (21) can be written as:

$$
A^{\mu}\left\{[\kappa](\varepsilon+\sigma)+(\sigma-\pi)\left[\tau_{1}\right] w^{-1} V^{\nu} B_{\nu}\right\}=0 .
$$

There is a second acoustic mode associated with propagation along $B^{\mu}$. The speed is:

$$
w_{2}=\frac{\pi-\sigma}{\varepsilon+\sigma} \frac{\left[\tau_{1}\right]}{[\kappa]} V^{\alpha} B_{\alpha} \quad W^{\mu}=A^{\mu}, V^{\mu} A_{\mu}=0 .
$$

2.3.2. $W^{\mu}=B^{\mu}$. The density variation, equation (18), for this case is:

$$
w^{2} \underline{\varepsilon}=-(\pi+\varepsilon)[\kappa] V^{\mu} A_{\mu} .
$$

The spatial discontinuities follow from equation (19) and are given by:

$$
\begin{aligned}
A^{\mu}\left\{[\kappa](\pi+\varepsilon)-\left[\tau_{1}\right](\sigma-\pi) w^{-1} V^{\alpha} B_{\alpha}\right\}+V^{\mu} \underline{\pi \varepsilon}+C^{\mu}\left[\tau_{2}\right](\sigma-\pi) w^{-1} V^{\alpha} B_{\alpha} \\
+B^{\mu}\left\{(\sigma-\underline{\pi}) \underline{\varepsilon} V^{\alpha} B_{\alpha}-\left[\tau_{1}\right](\sigma-\pi) V^{\alpha} A_{\alpha}+\left[\tau_{2}\right](\sigma-\pi) V^{\alpha} C_{\alpha}\right\}=0 .
\end{aligned}
$$

If the wave is propagating along $A^{\mu}$, so that $V^{\mu} A_{\mu}=1$, then we have:

$$
w_{3}=\sqrt{\pi} \quad\left[\tau_{1}\right]=0, V^{\mu} A_{\mu}=1 .
$$

If $V^{\mu}$ is not along $A^{\mu}$, then, as in the previous case, $\underline{\varepsilon}=0$ and there is no density variation. For this case we find a wave speed of:

$$
w_{4}=\frac{\sigma-\pi}{\varepsilon+\pi} \frac{\left[\tau_{1}\right]}{[\kappa]} V^{\alpha} B_{\alpha} \quad\left[\tau_{2}\right]=0, V^{\mu} A_{\mu} .
$$


2.3.3. $W^{\mu}=C^{\mu}$. The speed of sound for propagation along $A^{\mu}$ has the same form as (27). There is no restriction on $\left[\tau_{1}\right]$. For the case $V^{\mu} A_{\mu}=0$ there is no propagation, and we have $[\kappa]=\left[\tau_{2}\right]=0$.

\section{The amplitudes of the $W^{\mu}$ wave}

The amplitude of the $W^{\mu}$ wave, $\left|\underline{W^{\mu}}\right|$, can be used to further classify the various acoustic modes. Using equations (15)-(17) we can write the square amplitudes as:

$$
w^{2} \underline{W}^{\mu} \underline{W}_{\mu}=\left[\tau_{1}\right]^{2}-[\kappa]^{2} \quad \text { if } W^{\mu}=A^{\mu} .
$$

The longitudinal mode has $\left[\tau_{1}\right]=0$ and the amplitude is timelike. The complete $W^{\mu}$ vector develops a timelike part as the wave progresses through the medium. The transverse mode can be either spacelike, timelike, or null. The transverse mode is particularly interesting, since it occurs if $\left[\tau_{1}\right]= \pm[k]$. If initially the trajectory had no torsion or curvature, so that the developing discontinuity were to be the torsion and curvature, the equality of the torsion and curvature would be the condition for the curve to be a timelike helix [15]:

$$
w^{2} \underline{W}^{\mu} \underline{W}_{\mu}=\left[\tau_{1}\right]^{2}+\left[\tau_{2}\right]^{2} \quad \text { if } W^{\mu}=B^{\mu}
$$

$\underline{W}^{\mu}$ is a spacelike vector, and so $W^{\mu}$ remains a spacelike vector:

$$
w^{2} W^{\mu} \underline{W}_{\mu}=\left[\tau_{2}\right]^{2} \quad \text { if } W^{\mu}=C^{\mu}
$$

In the longitudinal mode $W^{\mu}$ remains a spacelike vector. There is no transverse mode for this case. The various amplitude behaviours along with the associated speeds of sound are summarized in table 1.

Table 1. Behaviours of amplitudes and associated speeds of sound.

\begin{tabular}{lllll}
\hline$W^{\mu}$ & $V^{\mu}$ & $w$ & $\underline{W^{\mu}}$ & Comment \\
\hline$A^{\mu}$ & $A^{\mu}$ & $\sqrt{\underline{\sigma}}$ & Timelike & Longitudinal $\left[\tau_{1}\right]=0$ \\
& $B^{\mu} \cdot C^{\mu}$ & $\frac{\pi-\sigma}{\varepsilon+\sigma} \frac{\left[\tau_{1}\right]}{[\kappa]} V^{\alpha} B_{\alpha}$ & Timelike & Transverse $\underline{\varepsilon}=0$ \\
& & Spacelike & \\
& & Null & Null case is timelike helix \\
\hline$B^{\mu}$ & $A^{\mu}$ & $\sqrt{\underline{\pi}}$ & Spacelike & Longitudinal $\left[\tau_{1}\right]=0$ \\
& $B^{\mu} \cdot C^{\mu}$ & $\frac{\sigma-\pi}{\varepsilon+\pi} \frac{\left[\tau_{1}\right]}{[\kappa]} V^{\alpha} B_{\alpha}$ & Spacelike & Transverse $\underline{\varepsilon}=0\left[\tau_{2}\right]=0$ \\
\hline$C^{\mu}$ & $A^{\mu}$ & $\sqrt{\underline{\pi}}$ & Spacelike & Longitudinal \\
& $B^{\mu}, C^{\mu}$ & & & {$[k]=0,\left[\tau_{2}\right]=0$}
\end{tabular}

\section{Discussion}

Taub [16] was the first to show that the relativistic speed of sound for a perfect fluid was simply the classical result $w=\sqrt{\underline{D}}=\sqrt{(\delta p / \delta \varepsilon)}$. The two longitudinal acoustic modes found for the anisotropic equivalent of the two perfect fluid system, $w_{1}=\sqrt{\delta \sigma / \delta \varepsilon}$ and $w_{3}=\sqrt{\delta \pi / \delta \varepsilon}$ are the intuitive extensions of Taub's result. These modes can be explained as the two fluids moving as a unit with the propagation direction parallel to the fluid acceleration $A^{\mu}$. The different values reflect a varying direction of anisotropy in the fluids. The other two transverse modes involve no net density variation, and should involve the motion of the two fluids relative to each other. In order to see how this occurs in the physical fluid, we need to briefly review Letelier's formalism. 


\subsection{Letelier Formalism}

The stress-energy tensor, equation (1), is invariant under the transformation:

$$
\begin{aligned}
& U_{\mu}^{*}=\cos (\alpha) U_{\mu 1}+\sin (\alpha) W_{\mu 2} \sqrt{\frac{p_{2}+\varepsilon_{2}}{p_{1}+\varepsilon_{1}}} \\
& W_{\mu}^{*}=\cos (\alpha) W_{\mu 2}-\sin (\alpha) U_{\mu 1} \sqrt{\frac{p_{1}+\varepsilon_{1}}{p_{2}+\varepsilon_{2}}} .
\end{aligned}
$$

Under this transformation equation (1) becomes:

$$
T_{\mu \nu}=\left(\varepsilon_{1}+p_{1}\right) U_{\mu}^{*} U_{\nu}^{*}+\left(\varepsilon_{2}+p_{2}\right) W_{\mu}^{*} W_{\nu}^{*}+\left(p_{1}+p_{2}\right) g_{\mu \nu}
$$

The angle $\alpha$ is chosen so that:

$$
U^{\mu *} W_{\mu}^{*}=0
$$

with $U^{\mu *}$ timelike and $W^{\mu *}$ spacelike. This angle is specified by:

$$
\tan (2 \alpha)=\frac{-2 U^{\mu}{ }_{1} W_{\mu_{2}} \sqrt{\left(p_{1}+\varepsilon_{1}\right)\left(p_{2}+\varepsilon_{2}\right)}}{p_{1}+\varepsilon_{1}-p_{2}-\varepsilon_{2}}
$$

Defining

$$
\begin{aligned}
U^{\mu} & =\frac{U^{\mu *}}{\sqrt{-U^{\alpha^{*}} U_{\alpha}^{*}}} \\
W^{\mu} & =\frac{W^{\mu *}}{\sqrt{W^{\alpha *} W_{\alpha}^{*}}}
\end{aligned}
$$

the stress-energy tensor becomes:

$$
T_{\mu \nu}=(\varepsilon+\pi) U_{\mu} U_{\mu}+(\sigma-\pi) W_{\mu} W_{\nu}+\pi g_{\mu \nu},
$$

which is equation (3) with

$$
\begin{aligned}
& (\varepsilon+\pi)=-\left(p_{1}+\varepsilon_{1}\right) U^{\mu *} U_{\mu}^{*} \\
& (\sigma-\pi)=\left(p_{2}+\varepsilon_{2}\right) W^{\mu *} W_{\mu}^{*} \\
& \pi=\left(p_{1}+p_{2}\right)
\end{aligned}
$$

or

$$
\begin{aligned}
& \varepsilon=\frac{\left(\varepsilon_{1}+\varepsilon_{2}-p_{1}-p_{2}\right)+H}{2} \\
& \sigma=\frac{-\left(\varepsilon_{1}+\varepsilon_{2}-p_{1}-p_{2}\right)+H}{2} \\
& H=\sqrt{\left(\varepsilon_{1}+p_{1}-\varepsilon_{2}-p_{2}\right)^{2}+4\left(U^{\mu}{ }_{3} W_{\mu 2}\right)^{2}\left(p_{1}+\varepsilon_{1}\right)\left(p_{2}+\varepsilon_{2}\right)}
\end{aligned}
$$

Note that equation (41) implies that $\sigma \geq \pi$. 


\subsection{The transverse modes}

If there is no density variation, i.e., $\underline{\varepsilon}=0$, then $\left[\pi_{, \mu}\right]=0$, and from (42) we have $\left[p_{1, \mu}\right]=-\left[p_{2, \mu}\right]$. The pressure variations in the two physical fluids are opposite. $\left[\sigma_{, \mu}\right]$ is also zero, and using (43) and (44) we find in addition that $\left[\varepsilon_{1, \mu}\right]=-\left[\varepsilon_{2, \mu}\right]$. The density variations in the two physical fiuids are also opposite to one another. The two fluids are moving relative to one another to maintain zero variation in pressure and energy density.

There are other examples of fluid systems that exhibit similar behaviour. One well known example is the speed of sound in superfluid helium [17]. In this system, first sound involves the normal fluid and the superfluid moving with the same speeds. In second sound, the two fluids move in opposition to each other with zero density change. Another example [18] is the constant density layer thickness oscillation in smetics.

\section{Application}

Van den Bergh and Skea [11] have discussed a cosmological metric of the form:

$\mathrm{d} s=-\mathrm{e}^{2(K+k)}(\mathrm{d} t)^{2}+\mathrm{e}^{+2(K+k)}(\mathrm{d} r)^{2}+\mathrm{e}^{2(S+s)}\left(\mathrm{e}^{-2(F+f)}(\mathrm{d} z)^{2}+\mathrm{e}^{+2(F+f)}(\mathrm{d} \phi)^{2}\right)$.

Using the two fluid stress-energy form described by (39), the five non-zero field equations are:

$$
\left(s^{\prime}\right)^{2}-\left(f^{\prime}\right)^{2}+2 k^{\prime} s^{\prime}-2 \ddot{S}+2 \dot{K} \dot{S}-3(\dot{S})^{2}-(\dot{F})^{2}=\sigma \mathrm{e}^{2(K+k)}
$$

$$
k^{\prime \prime}+s^{\prime \prime}+f^{\prime \prime}+\left(s^{\prime}+f^{\prime}\right)^{2}-\ddot{K}-\ddot{S}-\ddot{F}-(\dot{S}+\dot{F})^{2}=\pi \mathrm{e}^{2(K+k)}
$$

$(\phi \phi)$

$$
k^{\prime \prime}+s^{\prime \prime}-f^{\prime \prime}+\left(s^{\prime}-f^{\prime}\right)^{2}-\ddot{K}-\dot{S}+\ddot{F}-(\dot{S}-\dot{F})^{2}=\pi \mathrm{e}^{2(K+k)}
$$

$$
\begin{aligned}
& -2 s^{\prime \prime}+2 k^{\prime} s^{\prime}-3\left(s^{\prime}\right)^{2}-\left(f^{\prime}\right)^{2}+2 \dot{K} \dot{S}+(\dot{S})^{2}-(\dot{F})^{2}=\mu \mathrm{e}^{2(K+k)} \\
& \dot{S}\left(k^{\prime}-s^{\prime}\right)-f^{\prime} \dot{F}+s^{\prime} \dot{K}=0
\end{aligned}
$$

where we have assumed that the direction of anisotropy is in the radial direction. This would correspond to one of the two perfect fluids moving radially to the second. Van den Bergh and Skea, in their single perfect fluid treatment, assume an equation of state of the form $p=(\gamma-1) \varepsilon$, and discuss the solvability of the special case $\gamma=\frac{4}{3}$. We will use two equations of state:

$$
\sigma=n_{2} \varepsilon \quad \text { and } \quad \pi=n_{1} \varepsilon \text {, }
$$

with $n_{1}$ and $n_{2}$ the squares of the respective acoustic speeds for the anisotropic pressures $\pi$ and $\sigma$. Causality sets an upper limit of one for both speeds, and the formalism imposes the constraint:

$$
n_{1} \leq n_{2}<1
$$

The upper limit is not an equality, to avoid a singularity in $K$ in equation (56).

From the conservation laws for the system the energy can be determined. It is:

$$
\varepsilon=\varepsilon_{0} \exp \left[-\left(1+n_{1}\right) 2 S-\left(1+n_{2}\right) K\right] \exp \left[-k\left(\frac{1+n_{2}}{n_{2}}\right)-2 s\left(\frac{n_{2}-n_{1}}{n_{2}}\right)\right]
$$


Using the variables $X=s^{\prime}$ and $Y=\lambda s^{\prime}-f^{\prime}$ the field equations determine the anisotropic pressure $\sigma$ in terms of a function $\Phi=4 X^{2}-4\left(S_{0}\right)^{2}-Y^{2}$ :

$$
\sigma=\mathrm{e}^{-2(K+k)} \Phi(X, Y)
$$

The equation for energy (54) provides an integral constraint on $\Phi$. The field equations also provide the additional relations:

$$
\begin{array}{lll}
k=\lambda f+\frac{3-\lambda^{2}}{2} s & F=\lambda S & K=2\left(\frac{1+n_{1}}{1-n_{2}}\right) S \\
2 X^{\prime}+Y^{2}+\frac{\Phi}{n_{2}}=0 & Y^{\prime}+2 X Y+\lambda \Phi\left(\frac{1-n_{2}}{2 n_{2}}\right)=0 \\
\ddot{S}+2(\dot{S})^{2}-2\left(S_{0}\right)^{2}=0 &
\end{array}
$$

with

$$
\lambda^{2}=\frac{5+4 n_{1}-n_{2}}{1-n_{2}} .
$$

The time-dependent solutions to equation (58) are the same as discussed by Van den Bergh and Skea [11]. The two spatial equations (57) are the equations that admit an exact solution for $\gamma=\frac{4}{3}$ or $\lambda=3$ in the single fluid model. The same solution can be obtained in the two fluid model, but there is now a family of fluid parameters that correspond to this special solution. The family is described by the relations:

$$
n_{2}=\frac{\lambda-2}{\lambda} \quad n_{1}=\frac{[\lambda-(1-\sqrt{2})][\lambda-(1+\sqrt{2})]}{2 \lambda} .
$$

To maintain real speeds of sound and the constraint that $n_{2} \geq n_{1}, \lambda$ must lie between the limits:

$$
(1+\sqrt{2}) \leq \lambda \leq 3
$$

The lower limit corresponds to one dust $\left(n_{1}=0\right)$ and a second fluid $n_{1}=n_{2}=\frac{1}{3}$, equivalent to the original single fluid solution. Other solutions to the equations are under investigation. The solutions found by Van den Bergh and Skea generalize the solution of Feinstein and Senovilla [19]. The two fluid content that we describe would also apply to the Feinstein and Senovilla solution.

In conclusion, we have developed the acoustic mode speeds for a weakly discontinuous, isentropic two fluid system. The relationships are clearly of value in modelling and interpreting equations of state in general relativistic systems. The single perfect fluid content of the solution of Van den Bergh and Skea is extended to two fluids. Their solution corresponds to the comoving upper limit of two fiuids in relative motion to each other.

\section{Acknowledgment}

The authors would like to thank Professor F Hehl for reading the manuscript and for his very useful advice. 


\section{References}

[1] Coley A A and Tupper B O J 1984 Astrophys. J. 28026

[2] Coley A A and Tupper B O J 1986 J. Math. Phys. 27406

[3] Dunn K 1989 Gen. Rel. Grav. 21137

[4] Ferrando J J, Morales I A and Portilia M 1990 Gen. Ret. Grav. 221021

[5] Sistero R F 1971 Astrophys. Space Sci. 12484

[6] Mcintosh C B O 1968 Mon. Not. R. Astron. Soc. 140461

[7] Mcintosh C B O 1970 J. Math. Phys. 11250

[8] May T L and McVittie G C 1970 Mon. Not. R. Astron. Soc. 148407

[9] Bayin S S 1982 Phys. Rev. D 261262

[10] Letelier P S 1980 Phys. Rev. D 22807

[11] Van den Bergh $N$ and Skea J 1993 Class. Quantum Grav. 9527

[12] Carter B 1973 Phys. Rev. D 71590

[13] Lichnérowicz A 1971 Relativistic Hydrodynamics and Magnetohydrodynamics (New York: Benjamin)

[14] Anile A M 1989 Relativistic Fluids and Magneto-Fluids (Cambridge: Cambridge University Press) ch 4

[15] Synge J L 1960 Relativity: The General Theory (Amsterdam: North Holland) p 12

[16] Taub A H 1948 Phys. Rev. 74328

[17] Landau L D and Lifshitz E M 1986 Fluid Mechanics (Oxford: Pergamon Press) p 517

[18] Landau L D and Lifshitz E M 1986 Theory of Elasticity (Oxford: Pergamon Press) p 183

[19] Feinstein A and Senovilla J M M 1989 Class. Quantum Grav. 6 L89 